\title{
Low-energy absorption towards the ultra-compact X-ray binary 4U 1850-087 located in the globular cluster NGC 6712
}

\author{
L. Sidoli ${ }^{1}$, N. La Palombara ${ }^{1}$, T. Oosterbroek ${ }^{2}$, and A. N. Parmar ${ }^{3}$ \\ 1 Istituto di Astrofisica Spaziale e Fisica Cosmica “G. Occhialini”, IASF/INAF, via Bassini 15, 20133 Milano, Italy \\ e-mail: sidoli@iasf.mi.cnr.it \\ 2 Science Payload and Advanced Concepts Office, ESA, ESTEC, Postbus 299, 2200 AG, Noordwijk, The Netherlands \\ 3 Research and Scientific Support Department of ESA, ESTEC, Postbus 299, 2200 AG Noordwijk, The Netherlands
}

Received 16 May 2005 / Accepted 2 July 2005

\section{ABSTRACT}

We report the results of two XMM-Newton observations of the ultra-compact low-mass X-ray binary 4U 1850-087 located in the galactic globular cluster NGC 6712. A broad emission feature at $0.7 \mathrm{keV}$ was detected in an earlier ASCA observation and explained as the result of an unusual $\mathrm{Ne} / \mathrm{O}$ abundance ratio in the absorbing material local to the source. We find no evidence for this feature and derive $\mathrm{Ne} / \mathrm{O}$ ratios in the range $0.14-0.21$, consistent with that of the interstellar medium. During the second observation, when the source was $\sim 10 \%$ more luminous, there is some evidence for a slightly higher $\mathrm{Ne} / \mathrm{O}$ ratio and additional absorption. Changes in the $\mathrm{Ne} / \mathrm{O}$ abundance ratio have been detected from another ultra-compact binary, 4U 1543-624. We propose that these changes result from an X-ray-induced wind which is evaporated from an $\mathrm{O}$ and $\mathrm{Ne}$ rich degenerate donor. As the source X-ray intensity increases so does the amount of evaporation and hence the column densities and abundance ratio of $\mathrm{Ne}$ and $\mathrm{O}$.

Key words. accretion, accretion disks - stars: individual: 4U 1850-087 - stars: neutron Galaxy: globular clusters: individual: NGC 6712

\section{Introduction}

ASCA and BeppoSAX observations of ultra-compact $\left(P_{\text {orb }}<\right.$ $1 \mathrm{~h}$ ) low-mass X-ray binaries (LMXBs) have revealed two possible spectral differences compared to the longer period systems. These are (1) the presence of a discrete spectral feature near $0.7 \mathrm{keV}$ (attributed to $\mathrm{Ne}$ ) in the ASCA spectra (Juett et al. 2001) of X 1850-087, X 1543-624, X 0614+01 and X 0918-549 (the orbital periods of the last 2 sources are unknown, but their optical faintness is consistent with an ultra-compact nature) and (2) their best-fit parameter values when fitted with a disk-blackbody and Comptonized continuum (Sidoli et al. 2001). In the case of the ultracompact sources X 0512-401, X 1820-303, X 1850-087 and $\mathrm{X} 1832-330$, fits to the BeppoSAX spectra give significantly lower disk-blackbody temperatures than for other LMXB. In addition, the Comptonization seed photon temperatures appear consistent with those of the inner disk regions. The LMXBs located in the globular clusters NGC 6652 (Parmar et al. 2001) and Terzan 5 (Heinke et al. 2003) show very similar spectral properties, suggesting an ultra-compact nature for these binary systems (see Verbunt 2005 for a review).

The X-ray burst source X 1850-087 (Swank et al. 1976) is an X-ray binary located in the galactic globular cluster
NGC 6712, the least concentrated amongst those that host a luminous X-ray source. A short period (20.6 minutes) UV modulation was discovered with HST from the likely optical counterpart (Anderson et al. 1993), implying a degenerate companion of $0.04 M_{\odot}$ (Homer et al. 1996). The source is located $\sim 6^{\prime \prime}$ or $0.1 \pm 0.1$ core radii from the cluster center (Hertz \& Grindlay 1983). Another UV-excess star was discovered in the core of NGC 6712 with the ESO Very Large Telescope (Ferraro et al. 2000), a few arcsec away from the LMXB. The presence of these two interacting binaries inside the core of the low-density cluster NGC 6712 suggests that the interaction of the cluster with the disk and bulge of our Galaxy during numerous orbital passages plays a role in the formation of LMXBs in globular clusters (Ferraro et al. 2000). Moreover, there is evidence supporting a scenario where NGC 6712 was much more massive in the past and that it experienced a significant mass evaporation produced by the tidal force due to interactions with our Galaxy (see, e.g., Paltrinieri et al. 2001, and references therein).

EXOSAT observations of 4U 1850-087 revealed a complex spectrum. The best-fit was obtained with a model consisting of a power-law with a photon index, $\alpha$, of 0.4 with an exponential cut-off at $\sim 1 \mathrm{keV}$, together with a blackbody with a temperature, $k T$, of $2.4 \mathrm{keV}$ and absorption, $N_{\mathrm{H}}$, of $<5 \times 10^{21} \mathrm{~cm}^{-2}$ (Parmar et al. 1989). A thermal bremsstrahlung 
Table 1. XMM-Newton on-axis observation log of 4U 1850-087. Two observations were performed. The MOS1, MOS2 and pn cameras all used the medium thickness filter.

\begin{tabular}{llcccl}
\hline \hline Obs. & $\begin{array}{l}\text { Start time } \\
\text { (dy mon yr hr:mn) }\end{array}$ & $\begin{array}{c}\text { End time } \\
\text { (dy mon yr hr:mn) }\end{array}$ & Inst. & $\begin{array}{c}\text { Net Exp. } \\
(\mathrm{ks})\end{array}$ & Mode \\
\hline 1 & 27 Sep. 2003 09:05 & 27 Sep. 2003 12:22 & MOS1 & 11.7 & Timing Fast Uncompressed \\
1 & 27 Sep. 2003 09:05 & 27 Sep. 2003 12:22 & MOS2 & 11.5 & Imaging Prime Partial Window \\
1 & 27 Sep. 2003 09:10 & 27 Sep. 2003 12:23 & pn & 8.1 & Imaging Small Window \\
1 & 27 Sep. 2003 09:04 & 27 Sep. 2003 12:28 & RGS1 & 12.1 & Spectroscopy \\
1 & 27 Sep. 2003 09:04 & 27 Sep. 2003 12:28 & RGS2 & 12.1 & Spectroscopy \\
\hline 2 & 9 Oct. 2003 08:15 & 9 Oct. 2003 10:35 & MOS1 & 8.3 & Timing Fast Uncompressed \\
2 & 9 Oct. 2003 08:15 & 9 Oct. 2003 10:39 & MOS2 & 8.4 & Imaging Prime Partial Window \\
2 & 9 Oct. 2003 08:20 & 9 Oct. 2003 10:41 & pn & 5.9 & Imaging Small Window \\
2 & 9 Oct. 2003 08:14 & 9 Oct. 2003 10:43 & RGS1 & 8.8 & Spectroscopy \\
2 & 9 Oct. 2003 08:14 & 9 Oct. 2003 10:43 & RGS2 & 8.8 & Spectroscopy \\
\hline
\end{tabular}

$(k T=1.7 \mathrm{keV})$ absorbed by $5 \times 10^{21} \mathrm{~cm}^{-2}$ is a good approximation of the ROSAT Position Sensitive Proportional Counter spectrum (Verbunt et al. 1995). During a BeppoSAX survey of the bright LMRXB located in galactic globular clusters (Sidoli et al. 2001) the $0.3-50 \mathrm{keV}$ spectrum was fit with a disk-blackbody and Comptonized continuum with $N_{\mathrm{H}}=$ $3.9 \times 10^{21} \mathrm{~cm}^{-2}$, an inner disk temperature, $k T_{\text {in }}$, of $0.6 \mathrm{keV}$, an inner projected radius of $\sim 5 \mathrm{~km}$ (for an assumed NGC 6712 distance of $6.8 \mathrm{kpc}$, Harris 1996), a temperature, $k T_{0}$, of the input "seed" photons, of $0.8 \mathrm{keV}$ (consistent with the inner disk-blackbody temperature), an electron temperature, $k T_{\mathrm{e}}$, of $70 \mathrm{keV}$, and an optical depth, $\tau$, of 1.7 . The $0.1-100 \mathrm{keV}$ luminosity was $1.9 \times 10^{36} \mathrm{erg} \mathrm{s}^{-1}$.

Analysis of the ASCA Solid-state Imaging Spectrometer (SIS) data (Juett et al. 2001) from 4U 1850-087 revealed the presence of a spectral feature near $0.7 \mathrm{keV}$. A good fit to these data was found with an absorbed $k T=0.4 \mathrm{keV}$ blackbody together with a power-law with $\alpha=2.1$ when the relative abundances of $\mathrm{O}$ and $\mathrm{Ne}$ were allowed to vary. Both components are absorbed by $N_{\mathrm{H}}=2.9 \times 10^{21} \mathrm{~cm}^{-2}$, with a relative (to solar) $\mathrm{O} / \mathrm{H}$ abundance of $0.37 \pm 0.06$ and $\mathrm{Ne} / \mathrm{H}$ abundance of $1.9 \pm 0.3$. The authors interpreted this excess absorption as due to neutral Ne-rich material local to the binary. Preliminary results from the $0.4-2 \mathrm{keV}$ 4U 1850-087 XMM-Newton RGS spectra were reported in Sidoli et al. (2004), who found no evidence for an anomalous $\mathrm{Ne} / \mathrm{O}$ abundance ratio. Recently, analysis of Chandra LowEnergy Transmission Grating Spectrometer (LETGS) data confirmed this result (Juett \& Chakrabarty 2005), measuring a $\mathrm{Ne} / \mathrm{O}$ ratio of $0.22 \pm 0.05$ consistent with that expected from the interstellar medium (ISM) of 0.18 (Wilms et al. 2000).

Here we report the results of XMM-Newton observations performed in order to investigate the nature of the $0.7 \mathrm{keV}$ feature found with ASCA. We use the following updated values for the globular cluster NGC 6712 parameters (Paltrinieri et al. 2001): a distance of $8 \pm 1 \mathrm{kpc}$ (note that a previous estimate for the distance was $6.8 \mathrm{kpc}$, Harris 1996) and a reddening $E(B-V)=0.33 \pm 0.05$. Adopting the relation $A_{\mathrm{V}}=3.1 E(B-V)$, and $A_{\mathrm{V}}=N_{\mathrm{H}} \times 5.59 \times 10^{-22} \mathrm{~cm}^{-2}$
(Predehl \& Schmitt 1995) the optical reddening translates into an ISM column density of $(1.8 \pm 0.2) \times 10^{21} \mathrm{~cm}^{-2}$ to NGC 6712 .

\section{Observations}

The XMM-Newton Observatory (Jansen et al. 2001) includes three $1500 \mathrm{~cm}^{2} \mathrm{X}$-ray telescopes each with an European Photon Imaging Camera (EPIC) at the focus. Two of the EPIC imaging spectrometers use MOS CCDs (Turner et al. 2001) and one uses a pn CCD (Strüder et al. 2001). Behind two of the telescopes there are Reflection Grating Spectrometers (RGS, 0.35-2 keV; den Herder et al. 2001). XMM-Newton observed 4U 1850-087 twice, due to visibility problems, in 2003 September and October, about 12 days apart (see Table 1 for the observation details).

Data were reprocessed using version 6.1 of the Science Analysis Software (SAS). Known hot, or flickering, pixels and electronic noise were rejected using the SAS. The latest response matrices were used (updated to 2004-12-03, which should improve the agreement between the MOS and pn below $1 \mathrm{keV}$, Saxton 2004), while the ancillary response files were generated using the SAS task arfgen. Spectra were selected from single events only (pattern 0) for the MOS1 timing mode (only pattern 0 has been calibrated in this instrument mode) while for MOS2 patterns from 0 to 12 and for the pn patterns from 0 to 4 were selected. Source counts were extracted from circular regions of $40^{\prime \prime}$ radius centered on $4 \mathrm{U} 1850-087$ for the pn and the MOS2. With the SAS task epatplot we verified that pn Small Window data are not significantly affected by pile-up, whereas in both MOS spectra pile-up was evident. Thus, we minimized the effects of pile-up by extracting MOS 2 events in an annulus outside of a $10^{\prime \prime}$ radius core of the 4U 1850-087 point spread function, and MOS1 events from a wide column outside the central 15". A comparison between MOS1 and MOS2 spectra revealed that after this selection the source spectral shapes observed by the two instruments were similar. We use the pn for the determination of the source flux. Background counts were obtained from similar regions offset from the source position. The backgrounds do not show any 

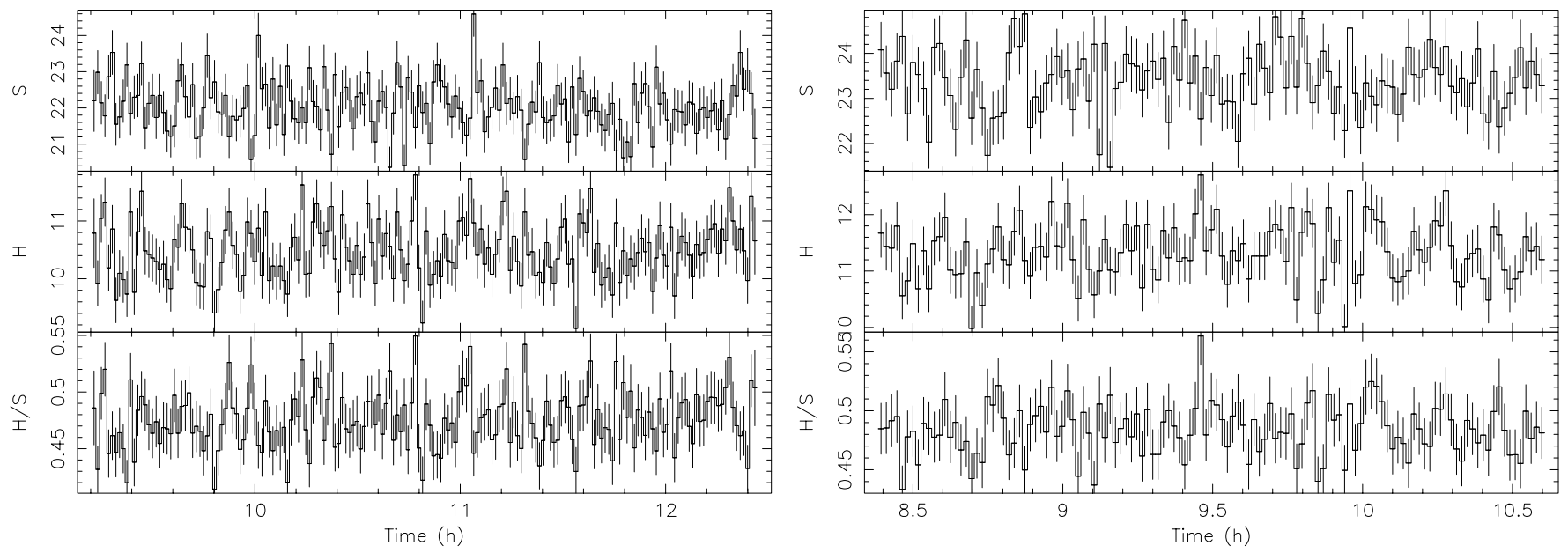

Fig. 1. EPIC pn lightcurves in two energy bands ( $\mathrm{S}, 0.3-2 \mathrm{keV}$ and $\mathrm{H}, 2-10 \mathrm{keV})$ and hardness ratios, $\mathrm{H} / \mathrm{S}$, during the first (left) and second observation (right). Time is in hours of 2003 September 27 (for the first observation) and of the 2003 October 9 (for the second observation). The binning is $64 \mathrm{~s}$.

evidence for flaring activity, so the entire nominal exposure times were considered. For both observations, the RGS spectra were analyzed as produced by the pipeline processing performed by XMM-Newton Survey Science Centre.

In order to ensure applicability of the $\chi^{2}$ statistic, the extracted spectra were rebinned such that at least 20 counts per bin were present and such that the energy resolution was not over-sampled by more than a factor 3 . Note that no systematic uncertainties were added to the spectra. All spectral uncertainties and upper-limits are given at $90 \%$ confidence for one interesting parameter.

\section{Results}

\subsection{Lightcurves}

Lightcurves for the two observations in soft $(0.3-2 \mathrm{keV})$ and hard $(2-10 \mathrm{keV})$ energy ranges were extracted in order to search for variability and hardness ratio variations (Fig. 2). The source was slightly harder and more intense during the second observation. Thus, the two observations were analyzed separately. Since within each individual observation the source does not show evidence for intensity or hardness variations (see Fig. 1), we can safely consider two separate spectra extracted from each of the two observations without making any further selections. The statistical quality of the data, combined with the length of the observations, does not allow for a meaningful search for periods around the optical period.

\subsection{Spectra}

We performed separate spectral analysis for the two XMM-Newton observations. We first studied the pn, MOS1 and MOS2 spectra in the energy range $0.3-12 \mathrm{keV}$. We noticed that the pn spectrum, in both the observations, showed a significant departure from the MOS1 and MOS2 shapes, especially around $0.6 \mathrm{keV}$, where a large excess is present only in the residuals of the pn spectrum (Fig. 3). A significant departure of the pn spectrum in this energy range is present also

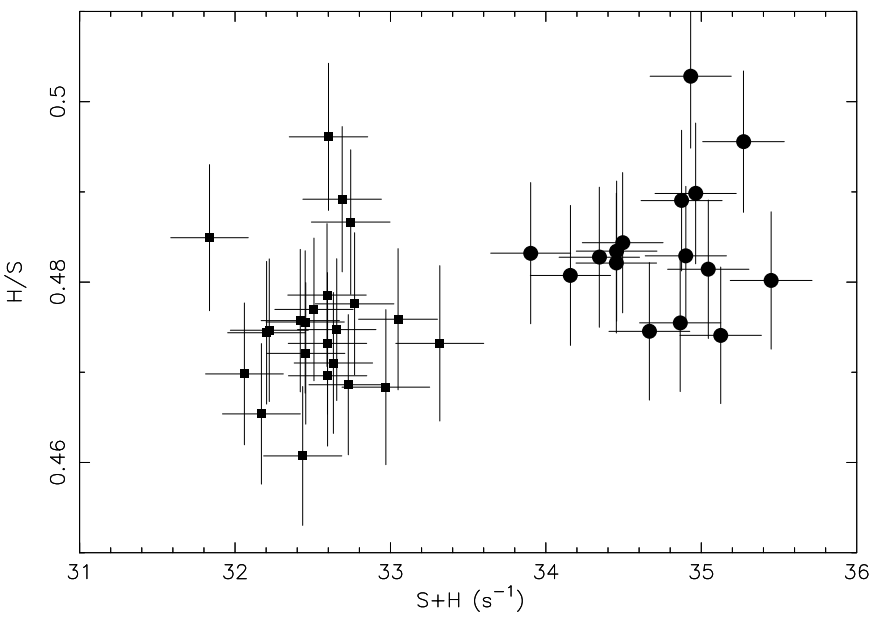

Fig. 2. Hardness ratio $(\mathrm{H}=2-10 \mathrm{keV} ; \mathrm{S}=0.3-2 \mathrm{keV}$ counts $)$ versus total intensity $(\mathrm{S}+\mathrm{H}, 0.3-10 \mathrm{keV})$ for the two pn observations (squares mark the first observation, circles the second). The binning is $512 \mathrm{~s}$.

compared with the RGS1 and RGS2. Note that this excess is not at the same energy of the feature present in the ASCA SIS spectra, which was interpreted as due to additional absorption by neutral Ne. Smaller differences between the pn and MOS cameras are also present at other energies, below $0.4 \mathrm{keV}$, and up to about $1.7 \mathrm{keV}$. Uncertainties in the calibration of the pn Small Window mode below $2 \mathrm{keV}$ are reported in Kirsch et al. (2004), although the use of the latest updated response matrices should reduce these differences (Saxton 2004). Examination of the pn background spectra does not reveal any features at these energies, so we are confident that they are not due to improper background subtraction. Moreover, both observations show similar shapes for the structured residuals. Thus, we restricted the pn energy range to $1.7-12 \mathrm{keV}$, where there are no significant differences between the MOS1, MOS2 and pn spectra.

In summary, we used the following energy ranges: $0.4-2 \mathrm{keV}$ for RGS1 and RGS2, $0.3-8 \mathrm{keV}$ for the MOS1 (because of the low statistics at high energy), $0.3-10 \mathrm{keV}$ for the 


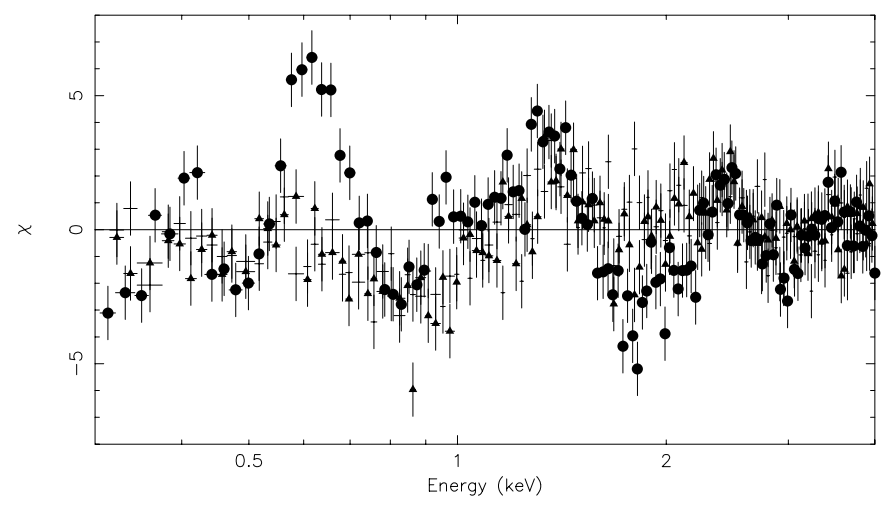

Fig. 3. Residuals (in units of standard deviations) when fitting the 2003 September MOS and pn spectra with a single absorbed power-law model. There is a different structure of the residuals in the pn (filled circles), compared with MOS1 (crosses) and MOS2 (triangles). A similar structured excess is present in the 2003 October spectrum.

MOS2, and 1.7-12 keV for the pn. The RGS spectra in both observations do not show evidence for edges or emission features. We investigated the $0.3-12 \mathrm{keV} 4 \mathrm{U} 1850-087$ spectra by simultaneously fitting the RGS1, RGS2, MOS1, MOS2 and pn spectra of each of the two observations individually. Factors were included in the spectral fitting to allow for normalization uncertainties between the instruments. In the spectral fitting XSPEC version 11.2 was used, and the interstellar abundances of Wilms et al. (2000) were used in the photoelectric absorption models.

LMXB X-ray spectra are generally fit with two component models, a black-body or a multicolor disk-blackbody component to account for the low-energy emission (originating from the accretion disk or the neutron star surface), and a highenergy component which is usually modeled with power-law, cut-off power-law, or Comptonized components, to account for the high-energy emission thought to be produced in a corona. In order to check if a soft component was required by the data we tried first with the simplest model consisting of an absorbed power-law (photoelectric absorption model PHABS in XSPEC). The fits resulted in positive residuals between $0.3-0.7 \mathrm{keV}$ and reduced $\chi^{2}=1.69$ for 1098 degrees of freedom (d.o.f.), and $\chi^{2}=1.94$ for 969 d.o.f. for the first and the second observations, respectively. Adding a blackbody improved the fits (reduced $\chi^{2}=1.51$ for 1096 d.o.f., and $\chi^{2}=1.78$ for 967 d.o.f.), but structured residuals at low-energies remain. Using a diskblackbody component instead of the blackbody resulted in better fits, with reduced $\chi^{2}=1.49$ for 1096 d.o.f., and $\chi^{2}=1.76$ for 967 d.o.f. for the first and second observations, respectively.

The absorption resulting from these fits was always higher than that derived from the optical reddening to NGC 6712. As well as the interstellar absorption in the direction of the globular cluster, modeled with PHABS with $N_{\mathrm{H}}$ fixed at $1.8 \times 10^{21} \mathrm{~cm}^{-2}$, we added another multiplicative component to investigate whether an ionized absorber could be present (ABSORI model in XSPEC). We fixed the Fe abundance of the absorber to the NGC 6712 value $([\mathrm{Fe} / \mathrm{H}]=-0.80 \pm 0.2)$, and linked the photon index of the ionizing continuum to that of the power-law component. The fit resulted in an un-ionized cold absorbing medium, with a best-fit ionization $\xi=L / n R^{2}$ in the range $0.5-2$ (where $L$ is the ionizing luminosity, $n$ the density of the absorbing medium, and $R$ is the distance of the obscuring material to the source). Thus, we do not consider further the presence of any ionized absorber.

We next tried partial covering (PCFABS model in XSPEC), absorbing both the disk-blackbody and power-law continuum components. We included a PHABS component with $N_{\mathrm{H}}$ fixed at $1.8 \times 10^{21} \mathrm{~cm}^{-2}$ to account for the interstellar absorption. The fit resulted in additional absorption in the range $6-8 \times 10^{21} \mathrm{~cm}^{-2}$ for the two observations, with a covering factor $\sim 95 \%$ for reduced $\chi^{2}=1.37$ for 1095 d.o.f., and $\chi^{2}=1.40$ for 966 d.o.f. for the first and the second observations, respectively. The diskblackbody temperatures were 0.6 and $0.3 \mathrm{keV}$ (with the innermost radii of the accretion disk $r_{\text {in }}(\operatorname{cosi})^{0.5}=3.9 \pm 0.5 \mathrm{~km}$ and $17 \pm 6 \mathrm{~km}$ ) while $\alpha=2.22 \pm 0.05$ and $2.35 \pm 0.02$ (with powerlaw normalizations of $0.060_{-0.008}^{+0.001}$ and $0.090 \pm 0.003$ photons $\mathrm{keV}^{-1} \mathrm{~cm}^{-2} \mathrm{~s}^{-1}$ at $1 \mathrm{keV}$, for the first and the second observations, respectively).

In order to investigate the $\mathrm{Ne} / \mathrm{O}$ abundance ratio, we used a variable abundance absorption model (VPHABS in XSPEC), with the elemental abundances set to the ISM values of Wilms et al. (2000) except for those of $\mathrm{O}, \mathrm{Ne}$ and $\mathrm{Fe}$ which were fixed to zero. Their absorption effect has been replaced with three edges ( $\mathrm{O}-\mathrm{K}, \mathrm{Fe}-\mathrm{L}$, Ne-K edges) with energies fixed at $0.54,0.71$ and $0.87 \mathrm{keV}$, and edge depths allowed to vary. In this way we could also account for a local iron abundance likely different from the cosmic value. We fit the spectra with 3 different two-components models for the continuum: (1) a disk-blackbody (DISKBB in XSPEC, Mitsuda et al. 1984) and a power-law, (2) a blackbody and a power-law, and (3) a disk-blackbody and a high-energy Comptonized component (COMPTT in XSPEC, Titarchuk 1994). This latter model was considered since it has been successfully fit to almost all the broad-band spectra of the galactic globular cluster LMXBs (Sidoli et al. 2001). Since the BeppoSAX best-fit electron temperature, $k T_{\mathrm{e}}$, of $\sim 70 \mathrm{keV}$ is well above the XMM-Newton upper energy threshold, it was fixed to $70 \mathrm{keV}$ for the fits performed here.

All 3 continuum models fit well and the best-fit continuum parameters are given in Table 2. All 3 models give similar $0.5-10 \mathrm{keV}$ source luminosities of $1.4 \times 10^{36} \mathrm{erg} \mathrm{s}^{-1}$ and $1.6 \times 10^{36} \mathrm{erg} \mathrm{s}^{-1}$ for a distance of $8 \mathrm{kpc}$ for the first and second observations, respectively. Table 3 gives the measured $\mathrm{Ne} / \mathrm{O}$ abundance ratios. These are all similar to the ISM value of 0.18 of Wilms et al. (2001). The equivalent column densities due to $\mathrm{O}$, Ne and Fe were then estimated by modeling the absorption as occurring from 3 edges with energies fixed at 0.54 , 0.71 and $0.87 \mathrm{keV}$ together with a narrow absorption line at an energy of $0.53 \mathrm{keV}$ (to account for O I ISM absorption). The resulting edge depths, columns and equivalent hydrogen columns are listed in Table 3 and shown in Fig. 4. In Fig. 5 we show the best fit spectra during the two observations.

As a final test, we tried fitting the spectra with the ASCA model, with the $\mathrm{Ne}$ and $\mathrm{O}$ abundances fixed at the ASCA best-fit values (Juett et al. 2001), letting all the other parameters vary in the usual way. We obtained unacceptable fits with reduced $\chi^{2}=2.0$ for 1096 d.o.f., and $\chi^{2}=2.2$ 
Table 2. Best-fit continuum parameters when the RGS1, RGS2, pn, MOS1 and MOS2 4U 1850-087 spectra were fit simultaneously. $d b b=$ diskblackbody, pow = power-law, $c t t=$ Comptonization model cOMPTT in XSPEC. $\alpha$ is the power-law photon index, $k T_{\text {in }}$ the inner disk temperature, $r_{\mathrm{in}}(\cos i)^{0.5}$ is the inner disk radius for a distance of $8 \mathrm{kpc}, i$ is the disk inclination angle and $R_{\mathrm{bb}}$ is the blackbody radius. For the comPTT model, $k T_{0}$ is the temperature of the "seed" photons, $k T_{\mathrm{e}}$ is the electron temperature (fixed at $70 \mathrm{keV}$ ) and $\tau_{\mathrm{p}}$ is the plasma optical depth.

\begin{tabular}{llllllllll}
\hline \hline Model & $\begin{array}{c}N_{\mathrm{H}} \\
\left(10^{21} \mathrm{~cm}^{-2}\right)\end{array}$ & $\alpha$ & $\begin{array}{c}k T_{\text {in }} \\
(\mathrm{keV})\end{array}$ & $\begin{array}{c}r_{\mathrm{in}}(\cos i)^{0.5} \\
(\mathrm{~km})\end{array}$ & $\begin{array}{c}k T_{\mathrm{bb}} \\
(\mathrm{keV})\end{array}$ & $\begin{array}{c}R_{\mathrm{bb}} \\
(\mathrm{km})\end{array}$ & $\begin{array}{c}k T_{0} \\
(\mathrm{keV})\end{array}$ & $\tau_{\mathrm{p}}$ & $\chi^{2} /$ d.o.f. \\
\hline $\begin{array}{l}\text { Observation } 1 \\
\text { dbb+pow }\end{array}$ & $5.7_{-0.2}^{+0.3}$ & $2.03_{-0.06}^{+0.06}$ & $0.69_{-0.02}^{+0.02}$ & $2.9_{-0.2}^{+0.2}$ & $\ldots$ & $\ldots$ & $\ldots$ & $\ldots$ & $1.34 / 1092$ \\
bb+pow & $6.1_{-0.2}^{+0.2}$ & $2.18_{-0.04}^{+0.04}$ & $\ldots$ & $\ldots$ & $0.48_{-0.02}^{+0.02}$ & $5.1_{-0.6}^{+0.6}$ & $\ldots$ & $\ldots$ & $1.37 / 1092$ \\
dbb+ctt & $4.1_{-0.1}^{+0.1}$ & $\ldots$ & $0.73_{-0.02}^{+0.03}$ & $2.5_{-0.2}^{+0.3}$ & $\ldots$ & $\ldots$ & $0.23_{-0.03}^{+0.01}$ & $1.2_{-0.1}^{+0.1}$ & $1.30 / 1091$ \\
\hline Observation 2 & & & & & & & & & \\
dbb+pow & $5.7_{-0.3}^{+0.2}$ & $2.16_{-0.06}^{+0.06}$ & $0.75_{-0.04}^{+0.06}$ & $1.8_{-0.4}^{+0.4}$ & $\ldots$ & $\ldots$ & $\ldots$ & $\ldots$ & $1.43 / 963$ \\
bb+pow & $5.9_{-0.2}^{+0.2}$ & $2.24_{-0.04}^{+0.04}$ & $\ldots$ & $\ldots$ & $0.55_{-0.04}^{+0.07}$ & $2.7_{-0.7}^{+1.0}$ & $\ldots$ & $\ldots$ & $1.44 / 963$ \\
dbb+ctt & $3.6_{-0.5}^{+0.2}$ & $\ldots$ & $0.84_{-0.05}^{+0.10}$ & $1.5_{-0.4}^{+0.4}$ & $\ldots$ & $\ldots$ & $0.22_{-0.02}^{+0.03}$ & $1.0_{-0.1}^{+0.1}$ & $1.38 / 962$ \\
\hline
\end{tabular}

Table 3. Photoelectric absorption results towards 4U 1850-087 (see Table 2 for the continuum emission parameters). The edge energies were fixed at $0.54,0.71$ and $0.87 \mathrm{keV}$ for $\mathrm{O}, \mathrm{Fe}$ and $\mathrm{Ne}$, respectively. $\tau_{\text {edge }}$ is the absorption depth. $N_{\mathrm{Z}}$ is the element column density (in units of $10^{17} \mathrm{~cm}^{-2}$ ) calculated using the Henke et al. (1993) cross sections. $N_{\mathrm{H}}$ is the hydrogen column density implied by $N_{\mathrm{Z}}$, in units of $10^{21} \mathrm{~cm}^{-2}$, assuming the ISM abundances of Wilms et al. (2000).

\begin{tabular}{|c|c|c|c|c|c|}
\hline Model & Edge & $\tau_{\text {edge }}$ & $N_{\mathrm{Z}}$ & $N_{\mathrm{H}}$ & $\mathrm{Ne} / \mathrm{O}$ \\
\hline \multicolumn{6}{|c|}{ Observation 1} \\
\hline $\mathrm{dbb}+$ & $\mathrm{Ne} \mathrm{K}$ & $0.19_{-0.05}^{+0.04}$ & $5.2_{-1.4}^{+1.1}$ & $6.0_{-1.6}^{+1.3}$ & \\
\hline \multirow[t]{2}{*}{ pow } & $\mathrm{OK}$ & $2.07_{-0.12}^{+0.12}$ & $36.4_{-2.1}^{+2.1}$ & $7.4_{-0.4}^{+0.4}$ & $0.14_{-0.04}^{+0.03}$ \\
\hline & $\mathrm{Fe} \mathrm{L}$ & $0.54_{-0.06}^{+0.06}$ & $0.77_{-0.09}^{+0.09}$ & $2.9_{-0.3}^{+0.3}$ & \\
\hline $\mathrm{bb}+$ & $\mathrm{Ne} \mathrm{K}$ & $0.20_{-0.04}^{+0.04}$ & $5.5_{-1.1}^{+1.1}$ & $6.3_{-1.3}^{+1.3}$ & \\
\hline \multirow[t]{2}{*}{ pow } & $\mathrm{OK}$ & $2.17_{-0.12}^{+0.12}$ & $38.2_{-2.1}^{+2.1}$ & $7.8_{-0.4}^{-0.3}$ & $0.14_{-0.03}^{+0.03}$ \\
\hline & $\mathrm{Fe} \mathrm{L}$ & $0.57_{-0.06}^{+0.06}$ & $0.81_{-0.09}^{+0.09}$ & $3.0_{-0.3}^{+0.3}$ & \\
\hline $\mathrm{dbb}+$ & $\mathrm{Ne} \mathrm{K}$ & $0.18_{-0.05}^{+0.04}$ & $5.0_{-1.1}^{+1.1}$ & $5.7_{-1.6}^{+1.3}$ & \\
\hline \multirow[t]{2}{*}{$+\mathrm{ctt}$} & $\mathrm{OK}$ & $1.76_{-0.13}^{+0.14}$ & $31.0_{-2.5}^{+2.5}$ & $6.3_{-0.5}^{+0.5}$ & $0.16_{-0.05}^{+0.04}$ \\
\hline & $\mathrm{Fe} \mathrm{L}$ & $0.46_{-0.06}^{+0.07}$ & $0.66_{-0.10}^{+0.10}$ & $2.4_{-0.3}^{+0.4}$ & \\
\hline \multicolumn{6}{|c|}{ Observation 2} \\
\hline $\mathrm{dbb}+$ & $\mathrm{Ne} \mathrm{K}$ & $0.27_{-0.05}^{+0.05}$ & $7.4_{-1.4}^{+1.4}$ & $8.5_{-1.6}^{+1.6}$ & \\
\hline \multirow[t]{2}{*}{ pow } & $\mathrm{OK}$ & $2.32_{-0.13}^{+0.13}$ & $40.8_{-2.3}^{+2.3}$ & $8.3_{-0.5}^{+0.5}$ & $0.18_{-0.04}^{+0.04}$ \\
\hline & $\mathrm{Fe} \mathrm{L}$ & $0.70_{-0.07}^{+0.07}$ & $1.0_{-0.1}^{+0.1}$ & $3.7_{-0.4}^{+0.4}$ & \\
\hline $\mathrm{bb}+$ & $\mathrm{Ne} \mathrm{K}$ & $0.29_{-0.05}^{+0.05}$ & $8.0_{-1.4}^{+1.4}$ & $9.2_{-1.6}^{+1.6}$ & \\
\hline \multirow[t]{2}{*}{ pow } & $\mathrm{OK}$ & $2.41_{-0.12}^{+0.12}$ & $42.4_{-2.1}^{+2.1}$ & $8.7_{-0.4}^{+0.4}$ & $0.19_{-0.03}^{+0.03}$ \\
\hline & $\mathrm{Fe} \mathrm{L}$ & $0.73_{-0.07}^{+0.07}$ & $1.0_{-0.1}^{+0.1}$ & $3.9_{-0.4}^{+0.4}$ & \\
\hline $\mathrm{dbb}+$ & $\mathrm{Ne} \mathrm{K}$ & $0.25_{-0.08}^{+0.04}$ & $6.9_{-2.2}^{+1.1}$ & $7.9_{-2.5}^{+1.3}$ & \\
\hline \multirow[t]{2}{*}{$\mathrm{ctt}$} & $\mathrm{OK}$ & $1.90_{-0.22}^{+0.14}$ & $33.5_{-3.9}^{+2.5}$ & $6.9_{-0.8}^{+0.5}$ & $0.21_{-0.07}^{+0.04}$ \\
\hline & $\mathrm{Fe} \mathrm{L}$ & $0.60_{-0.11}^{+0.07}$ & $0.86_{-0.16}^{+0.10}$ & $3.2_{-0.4}^{+0.4}$ & \\
\hline
\end{tabular}

for 967 d.o.f. with structured residuals evident below $1 \mathrm{keV}$ (see Fig. 6).

\section{Discussion}

We report the results of two XMM-Newton observations of 4U 1850-087 performed about 12 days apart. The spectra require a soft emission component, which is slightly better described by a multi-color disk-blackbody than a blackbody. At higher energies, a power-law provides a good fit the spectra. The photon index is similar to that measured during the ASCA observation (Juett et al. 2001), while it is significantly softer than during the later Chandra observation (Juett \& Chakrabarty 2005). This may be due to the fact that in fitting the Chandra LETGS spectrum the low-energy absorption was fixed to the ISM value towards the globular cluster. We note, however, that the source luminosities during the XMM-Newton and Chandra observations were similar, whilst during the ASCA observation the source was almost a factor 2 brighter with a $0.5-10 \mathrm{keV}$ luminosity of $2.5 \times 10^{36} \mathrm{erg} \mathrm{s}^{-1}$.

The total low-energy absorption resulting from the fits is similar in both the XMM-Newton observations and is $4-6.3 \times 10^{21} \mathrm{~cm}^{-2}$, depending on the model adopted for the continuum. There is evidence for extra-absorption in the line of sight, since the best-fit total $N_{\mathrm{H}}$ is always significantly higher than the optically derived value in the direction of the host globular cluster of $(1.8 \pm 0.2) \times 10^{21} \mathrm{~cm}^{-2}$. Thus the intrinsic absorption ranges from 2 to $4.5 \times 10^{21} \mathrm{~cm}^{-2}$, depending on the continuum model assumed. In the Comptonization model a lower column density is required because of the turnover at low energies present in the model. The presence of neutral extra-absorption local to the source is also confirmed by a good fit when using the partial covering fraction absorption model, which indicate that the central source is absorbed by a neutral medium with a covering factor of $\sim 95 \%$ and an intrinsic hydrogen column density in the range $6-8 \times 10^{21} \mathrm{~cm}^{-2}$ for the two observations.

We adopted a variable absorption model together with three edges (O-K, Ne-K and Fe-L) in order to measure the column density of the $\mathrm{Ne}, \mathrm{O}$ and $\mathrm{Fe}$ in the line of sight, since the ASCA spectrum suggests an excess absorption of neutral Ne-rich material local to the source (Juett et al. 2001). Other ultra-compact X-ray binaries $\left(P_{\text {orb }}<1 \mathrm{~h}\right)$ display overabundances of neutral Ne from the absorption effects in ASCA spectra. Moreover, during XMM-Newton and Chandra observations an anomalously high $\mathrm{Ne} / \mathrm{O}$ abundance ratio has been observed in a number of other ultra-compact binaries, 

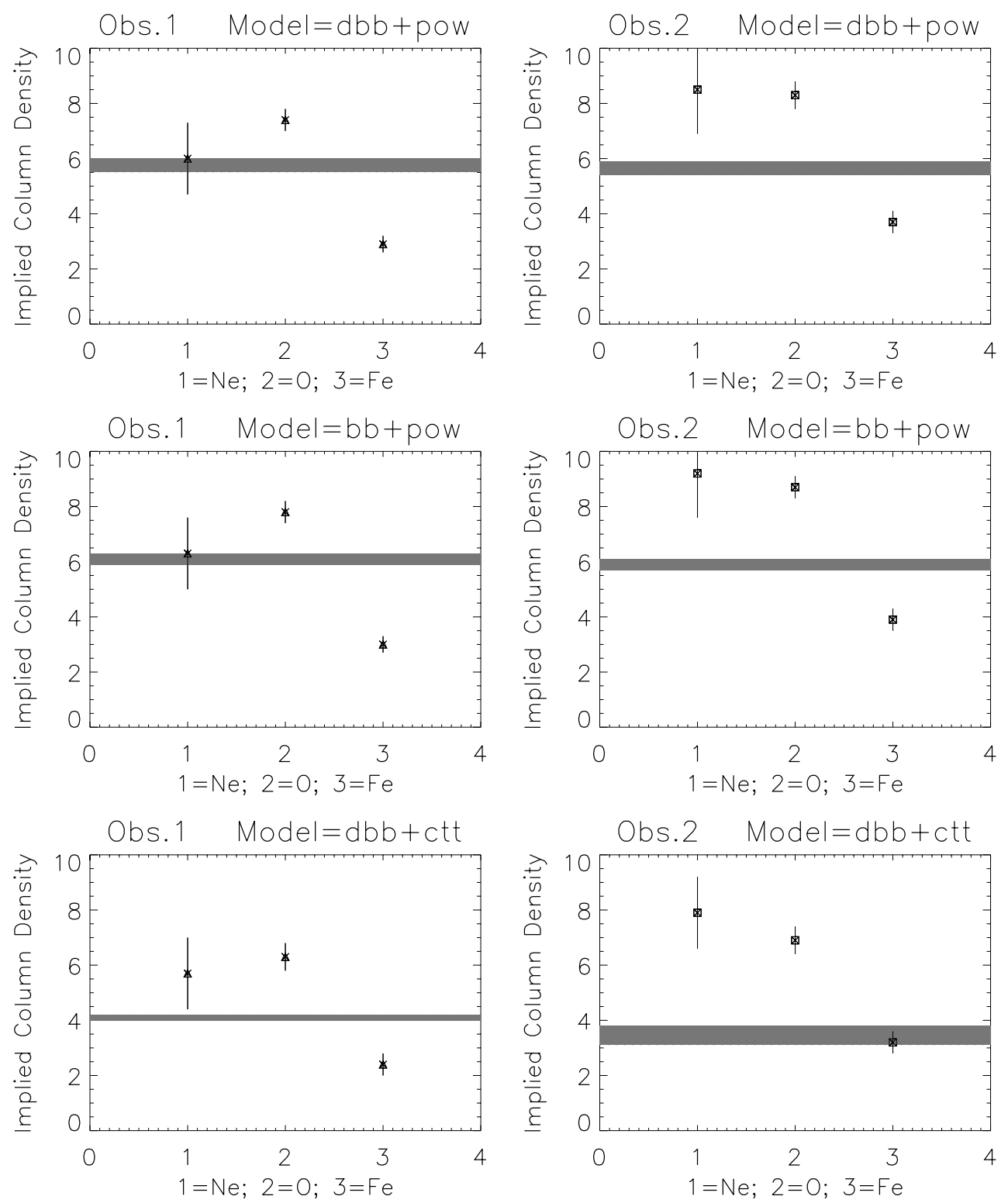

Fig. 4. The hydrogen column densities (in units of $10^{21} \mathrm{~cm}^{-2}$ ) implied by the optical depths of the $\mathrm{Ne}, \mathrm{O}$ and Fe edges, from the first (left) and second observations (right), depending on the different models assumed for the continua (Table 3). Shadowed regions mark the $90 \%$ confidence range for $N_{\mathrm{H}}$ resulting from the continuum fits (VPHABS model, see Table 2).

indicative of neutral Ne overabundance (e.g., 4U 0614+091, Paerels et al. 2001; 4U 1543-624 and 2S 0918-549, Juett \& Chakrabarty 2003). Thus, it has been proposed that the donor stars in some ultra-compact binary systems are Ne-rich white dwarfs (e.g., Yungelson et al. 2002; Bildsten 2002).

The high resolution RGS 4U 1850-087 spectra presented here do not show any prominent emission features or other absorption edges, besides those of O-K, Ne-K and Fe-L. Our study of the absorbing Ne and O toward 4U 1850-087 reveals an $\mathrm{Ne} / \mathrm{O}$ abundance ratio (see Table 3 ) which is consistent with the ISM value of 0.18 (Wilms et al. 2000). This result is in agreement with the Chandra observation performed in 2002 (Juett \& Chakrabarty, 2005) but contrary to the earlier ASCA measurement (Juett et al. 2001).

If the measured elemental column densities $N_{\mathrm{Z}}$ (see Table 3) are converted to equivalent $\mathrm{H}$ column densities using the Wilms et al. (2000) ISM abundances, we can compare the $N_{\mathrm{H}}$ resulting from the overall shape of the XMMNewton spectra (dashed regions in Fig. 4 include the $90 \%$ uncertainties on the $N_{\mathrm{H}}$ resulting from the fit). For each continuum model, for both observations, there seems to be a discrepancy between the total $N_{\mathrm{H}}$ and the equivalent $N_{\mathrm{H}}$ calculated from the elemental column densities $N_{Z}$. This could indicate an over-abundance of $\mathrm{Ne}$ and $\mathrm{O}$ and a sub-solar abundance of $\mathrm{Fe}$. This under-abundance may be explained by the low Fe abundance of the host globular cluster. Alternatively, it is possible that the uncertainties on the derived hydrogen column densities could be underestimated. Indeed, Paerels et al. (2000) point out that the photoelectric cross-sections could have a $30 \%$ uncertainty which is large enough to account for this discrepancy.

There is no evidence for any temporal variability of the $\mathrm{Ne}$, $\mathrm{O}$ and Fe column densities between the two XMM-Newton 

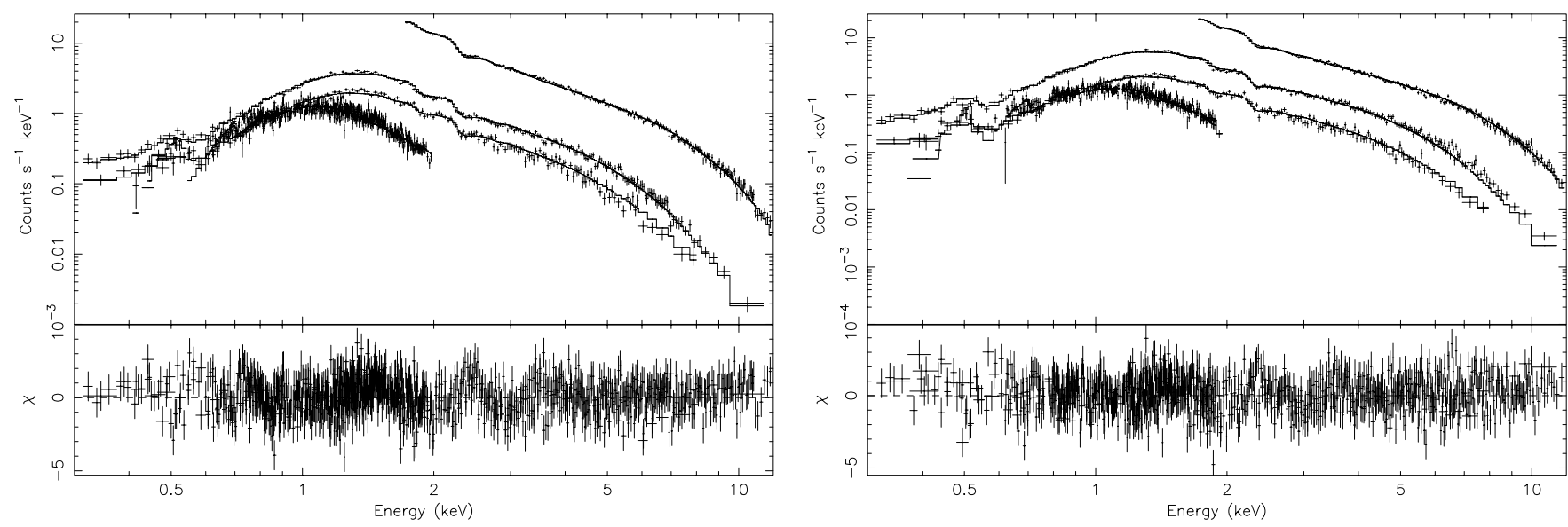

Fig. 5. The 0.3-12 keV 4U 1850-087 count spectra (together with the residuals in units of standard deviation) from the two observations (left panel shows the first observation, the right panel the second). The continua have been modeled with a disk-blackbody and a power-law (see Tables 2 and 3 for the continuum and line parameters, respectively).
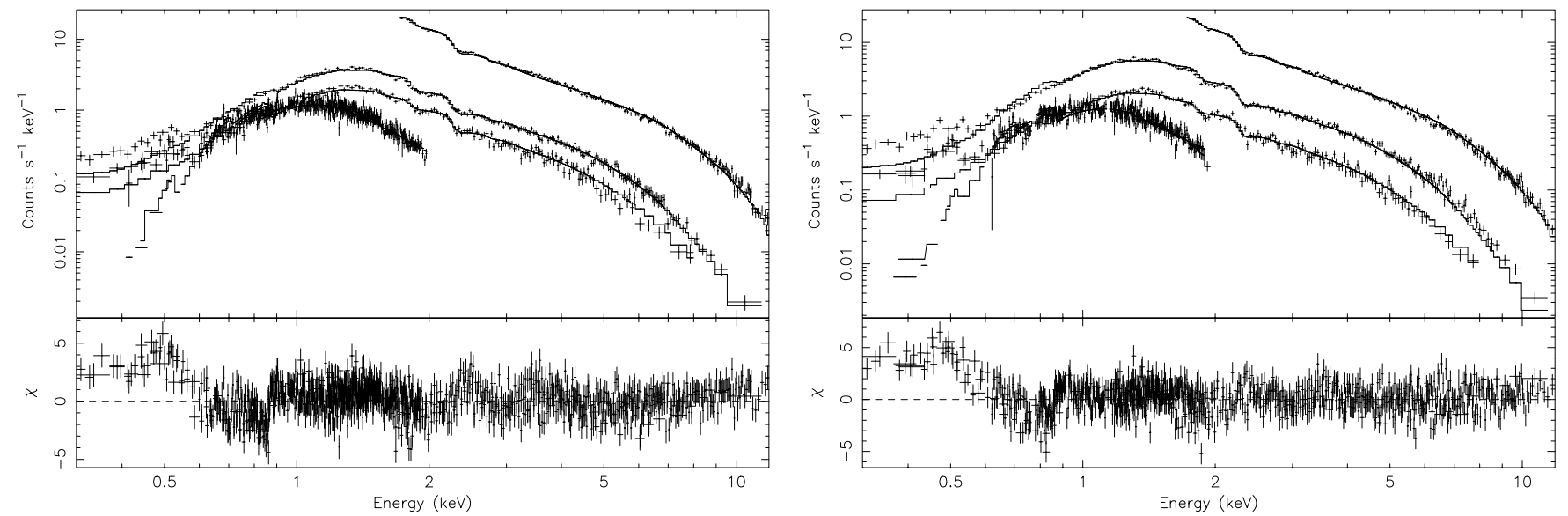

Fig. 6. The $0.3-12 \mathrm{keV} 4 \mathrm{U} 1850-087$ count spectra (together with the residuals in units of standard deviations) from the two observations (left panel shows the first observation, the right panel the second), fit with the ASCA model with the Ne and O abundances fixed at the values reported in Juett et al. (2001).

observations (within $90 \%$ uncertainty), although we note that the column densities are systematically higher in the second observation. Also the $\mathrm{Ne} / \mathrm{O}$ ratio, although always compatible within uncertainties with the standard ISM value, is on average higher during the second observation. The 4U 1850-087 spectrum during the two XMM-Newton observations differs in the total $0.5-10 \mathrm{keV}$ luminosity (see Table 2) with the second observation being $\sim 10 \%$ more luminous than the first. This suggests a possible correlation between the $\mathrm{Ne} / \mathrm{O}$ abundance ratio and the X-ray source luminosity, and a possible explanation for the different $\mathrm{Ne} / \mathrm{O}$ ratios observed with XMM-Newton, Chandra, and ASCA. Note that during the ASCA observation, where evidence for a strong $\mathrm{Ne}$ overabundance was reported, the source luminosity was almost twice that during the XMM-Newton and Chandra observations.

Juett \& Chakrabarty (2003) proposed that ionization could play a role in the variable abundance ratios. We suggest another possible mechanism which could help in the understanding of the local extra-absorption and its metal abundance. Maccarone et al. (2004) studied the irradiation-induced stellar winds in $\mathrm{X}$-ray binaries in order to explain different X-ray spectra from
LMXBs located in globular clusters with different metallicities. An evaporative wind can be produced even in LMXBs with degenerate companions, where part of the radiation produced from the central source illuminates the donor star (e.g., Ruderman et al. 1989). This wind could contribute to the observed column density toward the X-ray source, leading to an intrinsic column density of $\sim 6 \times 10^{21} \mathrm{~cm}^{-2}$ (see Eq. (7) in Maccarone et al. 2004). During the XMM-Newton observations we found a comparable amount of extra-absorption towards $4 \mathrm{U} 1850-087$. We suggest that a wind evaporated from the degenerate companion could be responsible for the intrinsic absorption observed. Using the 4U 1850-087 observed parameters, and Eq. (7) of Maccarone et al. (2004), we derive a wind velocity of $\sim 5 \times 10^{7} \mathrm{~cm} \mathrm{~s}^{-1}$, which may be confined to the binary (the escape velocity is $\sim 10^{8} \mathrm{~cm} \mathrm{~s}^{-1}$ ). A higher source luminosity would translate into a larger contribution by the wind from the degenerate donor, which is likely to be rich in $\mathrm{Ne}$ and $\mathrm{O}$. We suggest that this mechanism could contribute to the different abundance ratio observed from 4U 1850-087 with XMM-Newton, Chandra, and ASCA. This could possibly help in explaining why during higher source luminosity 
intervals, higher $\mathrm{Ne} / \mathrm{O}$ abundance ratios are observed. We note that another ultra-compact X-ray binary, 4U 1543-624, displays a variable $\mathrm{Ne} / \mathrm{O}$ abundance ratio. Juett \& Chakrabarty (2003) measured an $\mathrm{Ne} / \mathrm{O}$ abundance ratio of $1.5 \pm 0.3$ with Chandra, and $0.54 \pm 0.03$ with XMM-Newton. The higher $\mathrm{Ne} / \mathrm{O}$ abundance ratio was observed when the source was more luminous, as appears to be the case with $4 \mathrm{U}$ 1850-087.

Theoretical models for the formation of white dwarfs predict a Ne/O abundance ratio in the range 0.2-0.4 (e.g., Deloye \& Bildsten 2002; Segretain et al. 1994; Gutierrez et al. 1996), which is low compared with the $\mathrm{Ne} / \mathrm{O}$ ratios observed with ASCA in 4U 1850-087 (or with Chandra in 4U 1543-624). On the other hand, Yungelson et al. (2002), studying the formation of Ne-enriched donors in ultracompact X-ray binaries, point out that the abundance of neon in the nucleus of the dwarf may be underestimated by a factor of 3 (Isern et al. 1991), which makes the theoretically predicted $\mathrm{Ne} / \mathrm{O}$ ratios agree better with the higher observed values.

Acknowledgements. Based on observations obtained with XMM-Newton, an ESA science mission with instruments and contributions directly funded by ESA member states and the USA (NASA).

\section{References}

Anderson, S. F., Margon, B., Deutsch, E. W., \& Downes, R. A. 1993 , AJ, 106, 1049

Bildsten, L. 2002, ApJ, 577, L27

Deloye, C. J., \& Bildsten, L. 2002, ApJ, 580, 1077

Den Herder, J. W., Brinkman, A. C., Kahn, S. M., et al. 2001, A\&A, 365, L7

Ferraro, F. R., Paltrinieri, B., Paresce, F., \& De Marchi, G. 2000, ApJ, 542, L29

Frank, J., King, A. R., \& Lasota, J.-P. 1987, A\&A, 178, 137

Gutierrez, J., Garcia-Berro, E., Iben, I., et al. 1996, ApJ, 459, 701

Harris, W. E. 1996, AJ, 112, 1487

Heinke, C., Edmonds, P., Grindlay, J., et al. 2003, ApJ, 590, 809
Henke, B. L., Gullikson, E. M., \& Davis, J. C. 1993, Atomic Data \& Nucl. Data Tables, 54, 181

Hertz, P., \& Grindlay, J. E. 1983, ApJ, 275, 105

Homer, L., Charles, P. A., Naylor, T., et al. 1996, MNRAS, 282, L37

Isern, J., Hernanz, M., Mochkovitch, R., et al., 1991, A\&A, 241, L29

Jansen, F., Lumb, D., Altieri, B., et al. 2001, A\&A, 365, L1

Juett, A. M., \& Chakrabarty, D. 2003, ApJ, 599, 498

Juett, A. M., \& Chakrabarty, D. 2005, ApJ, 627, 926

Juett, A. M., Psaltis, D., \& Chakrabarty, D. 2001, ApJ, 560, L59

Kirsch, M. G. F., Altieri, B., Chen, B., et al. 2004, http://xmm.vilspa.esa.es/docs/documents/

CAL-TN-0055-1-0.ps.gz, [arXiv:astro-ph/0407257]

Maccarone, T. J., Kundu, A., \& Zepf, S. E. 2004, ApJ, 606, 430

Mitsuda, K., Inoue, H., Koyama, K., et al. 1984, PASJ, 36, 741

Paerels, F., Brinkman, A. C., van der Meer, R. L. J., et al. 2001, ApJ, 546, 338

Parmar, A. N., Stella, L., \& Giommi, P. 1989, A\&A, 222, 96

Parmar, A. N., Oosterbroek, T., Sidoli, L., et al. 2001, A\&A, 380, 490

Predehl, P., \& Schmitt, J. H. M. M. 1995, A\&A, 293, 889

Paltrinieri, B., Ferraro, F. R., Paresce, F., et al. 2001, AJ, 121, 3114

Ruderman, M., Shaham, J., Tavani, M., et al. 1989, ApJ, 343, 292

Saxton, R. D. 2004, XMM-Newton CCF Release Note 183, http://xmm.vilspa.esa.es/docs/documents/ CAL-SRN-0183-1-0.ps.gz

Segretain, L., Chabrier, G., Hernanz, M., et al. 1994, ApJ, 434, 641

Sidoli, L., Parmar, A. N., Oosterbroek, T., et al. 2001, A\&A, 368, 451

Sidoli, L., Parmar, A. N., \& Oosterbroek, T. 2004, Proc. of the V INTEGRAL Workshop, ESA SP-552, 389

Strüder, L., Briel, U., Dennerl, K., et al. 2001, A\&A, 365, L18

Swank, J. H., Becker, R. H., Pravdo, S. H., et al. 1976, IAU Circ., 3010

Titarchuk, L. 1994, ApJ, 434570

Turner, M. J. L., Abbey, A., Arnaud, M., et al. 2001, A\&A, 365, L27

Verbunt, F. 2005, Proc. of Interacting binaries, July 4-10 Cefalu, ed. L. Antonelli, et al., to be published with AIP [arXiv: astro-ph/0412524]

Verbunt, F., Bunk, W., Hasinger, G., \& Johnston, H. M. 1995, A\&A, 298, 21

Wilms, J., Allen, A., \& McCray, R. 2000, ApJ, 542, 914

Yungelson, L. R., Nelemans, G., \& van den Heuvel, E. P. J. 2002, A\&A, 388, 546 